\title{
The Respiratory Prosody of Seven-Syllable Modern-Style Poems Chanted in Cantonese*
}

\author{
HOU Xing-quan, DENG De-chong, YANG-Feng \\ Jinan University, Guangzhou, China
}

\begin{abstract}
The paper focuses on the respiratory prosody of seven-syllable modern-style poems chanted in Cantonese, especially the relationship between respiratory prosody and sentential pause-extension. By collecting and analyzing respiratory and acoustic-phonetic signals simultaneously, this research reveals a two level chest and abdominal breath reset in the respiratory signals: (1) In the first level (L1), the breath reset appears at the beginning or the end of the clause. (2) In the second level (L2), the breath reset appears in the middle of the clause, of which the position relates to different tone patterns. There is a strong connection between respiratory prosody and the sentential pause-extension when chanting the seven-syllable modern-style poems in Cantonese: with L1 breath reset corresponding to the longest pause-extension at the end of the clause, while L2 breath reset corresponding to the longer pause-extension in the middle of the clause, usually falling at the end of the second syllable of the clause with tone pattern of Level-start and Level-end, or at the end of the fourth syllable of the clause with other tone patterns.
\end{abstract}

Keywords: poem, chanting, respiratory, prosody, pause-extension

\section{Introduction}

Respiration plays a vital role on human survival and verbal communication. At present, the physiological study of speech has become an important aspect of the study of phonetics. By sorting out the published works related to speech production, it is easy to find that there is more research about voice, resonance and lip, while less research on the respiratory prosody of speech. At an early stage, the research about respiration focused on physiological mechanism of respiration, such as the studies of Ron J. Baken et al (1979) and Thomas J. Hixon (1987). Since entering the 21st century, more studies about respiratory of speech began to spring up. Slifka J. (2000) described the respiratory system dynamics at the initiation termination of utterances and determines correlations of physiological measures with acoustic cures for these prosodic boundaries. Yuan Chu, Li Aijun (2007) studied the abnormal pause in the speech corpus and dialogue corpus, and focused its influence on the acoustic characteristics of the front and back prosodic phrases. Shi Feng et al (2010) found that the subsequent quantitative analysis and interpretation of the experiment recordings reveal the difference between respiratory movements in speech and in natural state. Unlike natural respiration, every respiratory parameter in speech is closely related to the prosodic hierarchy. Zhang Jinyu, Shi Feng and Bai Xuejun (2011) analyzed the difference

\footnotetext{
* Acknowledgements: This paper is supported by the innovation fund of Jinan University (No: 15JNQM024). HOU Xing-quan, Doctor, Associate Professor, Department of Chinese Language and Literature, Jinan University. DENG De-chong, Master Degree Candidate, Department of Chinese Language and Literature, Jinan University. YANG-Feng, Doctor, Associate Professor, Institute of Applied Linguistics, Jinan University.
} 
of respiratory rhythms in the states of narrating and reading. Zhang Jinyu (2011) showed some interesting results in her research: (1) when speech pause time grows, rhythmic unit is bigger; (2) speech pause duration and respiratory reset duration have positive correlations; (3) speech pause duration and respiratory reset amplitude also have positive correlations; (4) speech pause duration and respiratory reset slope have negative correlations. Yang Feng (2012) systematically studied the traditional poem chanting of four speakers from Fuzhou, Wenzhou, Changzhou, Jiangyin with four different signals collected simultaneously, and developed a corresponding speech respiratory prosody analysis platform. Yang' research laid a good foundation for the research of this paper both in theory and in method.

Overall, the previous research mainly focused on exploring Mandarin reading respiratory prosody rules, while little research was involved in the characteristics of different Chinese dialects of the respiratory prosody. Yang Feng (2012) mainly focused on the general rules of traditional dialect chanting, but the characteristics of chanting in different dialects were not discussed and less attention was paid to the relationship between respiratory prosody and phonetics prosody when chanting.

Chanting poems is a traditional way to read out poems with prolonged sounds and characteristic melody, the phonetic form of chanting is between singing and reading. Zhao Yuanren (1994) ever gave a clear definition of chanting: Chanting in China, is roughly according to the character of the tone to create a melody of improvisation, rather than strictly follow the tone to produce an unchanging melody. To a better understanding of the respiratory and phonetic features of traditional chanting in Chinese dialects, we take the seven-syllable modern-style poems chanted in Cantonese as an example, especially focus on the relationship between its phonetics prosody and respiratory prosody, and summarize the characteristic of its rhythm and prosody.

\section{Experimental Design}

\section{Experimental Speech Materials and Speakers}

More than thirty different seven-syllable poems of modern-style were chosen as the experimental speech materials. Only three men aged more than sixty were chosen as experimental informants, for people who are familiar with traditional Cantonese chanting are hard to find. All the informants come from Cantonese Speaking Areas, so they can speak Cantonese very fluently. They are healthy, articulate, with good style of chanting and classical cultural literacy, and two of them also have old-style private education experience.

\section{Experimental Equipment and Signal Analysis}

Microphone, sound console, electroglottography (EGG), respiratory belt transducer, 16 channel collector (Power Lab) and chart 7 were mainly employed to collect chest and abdominal respiratory signals and phonetic signals simultaneously. The analyzing platform named Speech Prosody and Respiration Analysis (SPRA for short hereafter) is developed by Yang Feng, who is also one of the authors of this paper.

All the signals were labeled and extracted parameters by SPRA, the working interface of SPRA is showed in Figure 1. Five signals in five sub-windows are displayed in SPRA. The five signals, from top to bottom, are: (1) speech signal, (2) EGG signal (unused in this research), (3) chest breathing signal, (4) abdominal breathing,

(5) the sum of chest and abdominal breathing. On the right of the interface, there are four functional plots and in each of them, some functional buttons are used for text labeling and signal processing. 


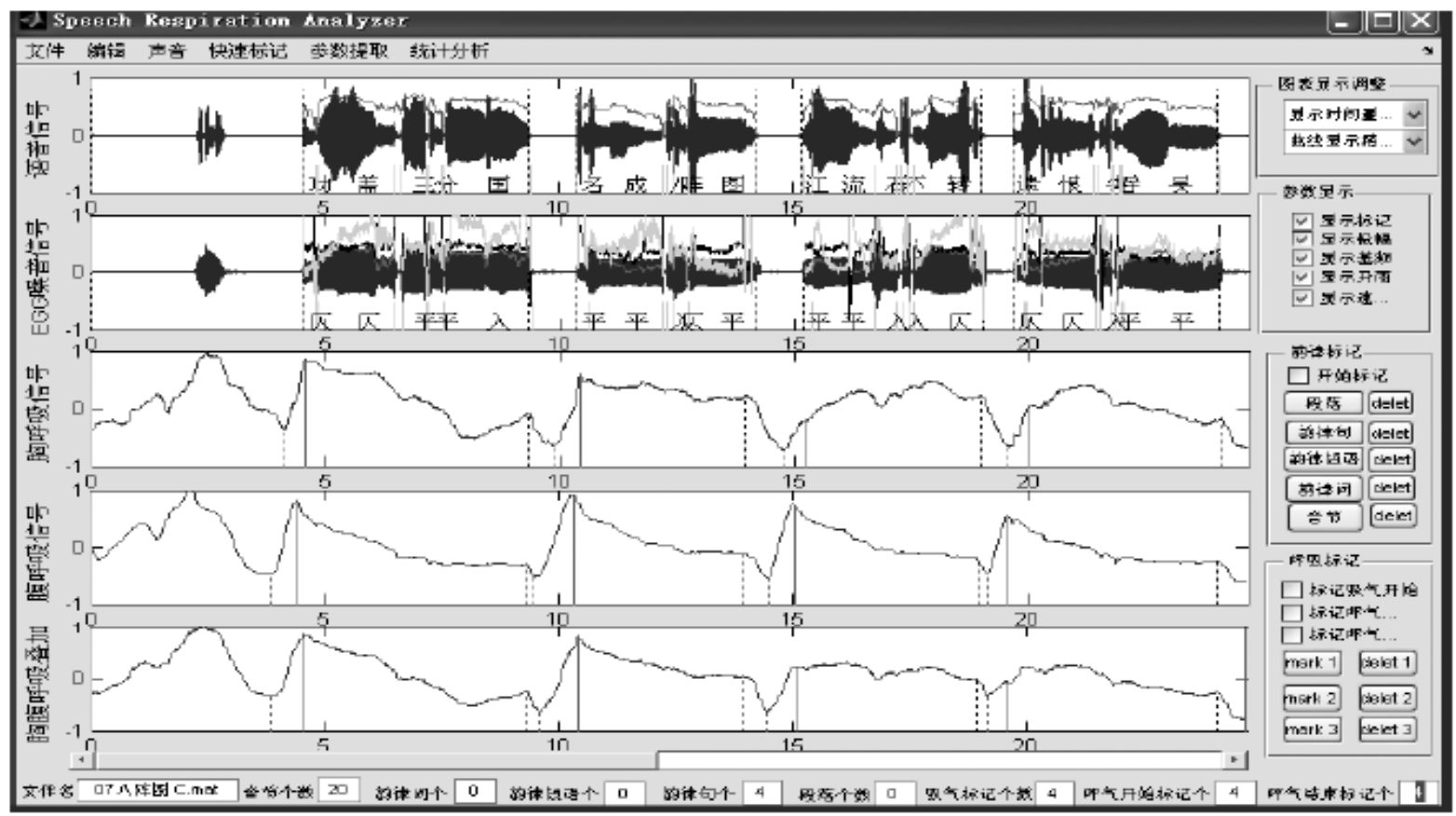

Figure 1. The interface of SPRA.

The prosodic boundaries of each layer are marked manually. While the inhalation onset time, breathing onset time, and exhalation end time of each period of chest and abdominal breathing are marked automatically. The breathing parameters such as the duration of inhalation (TI), duration of exhalation (TE), inhalation reset amplitude (AI), exhalation reset amplitude (AE), can be calculated by SPRA. Other breathing parameters, like the slope of inhalation period, slope of exhalation period, area of inhalation phase, and area of breathing phase, can also be measured with those parameters mentioned above. The definition of these breathing parameters was introduced by Yang Feng (2015). The parameters are saved as the format of .mat which can be analyzed by SPRA. The final breathing parameters are saved in Microsoft Excel for statistical analysis.

\section{Experimental Analysis and Results}

\section{Respiratory Prosodic Feature of Seven-Syllable Modern-Style Poems Chanted in Cantonese}

Generally speaking, both chest and abdominal respiration are used simultaneously when chanting seven-syllable poems, so the signal correlation between chest and abdominal respiration is very obvious, although the magnitude of chest and abdominal respiration may be different from different people, some informants may use chest respiration more than abdominal respiration, while others prefer to use abdominal respiration. In order to eliminate this kind of interpersonal difference, this research mainly takes the sum of chest and abdominal breathing reset (channel 5 signal) as a key parameter to distinguish the hierarchy of respiratory prosody. 


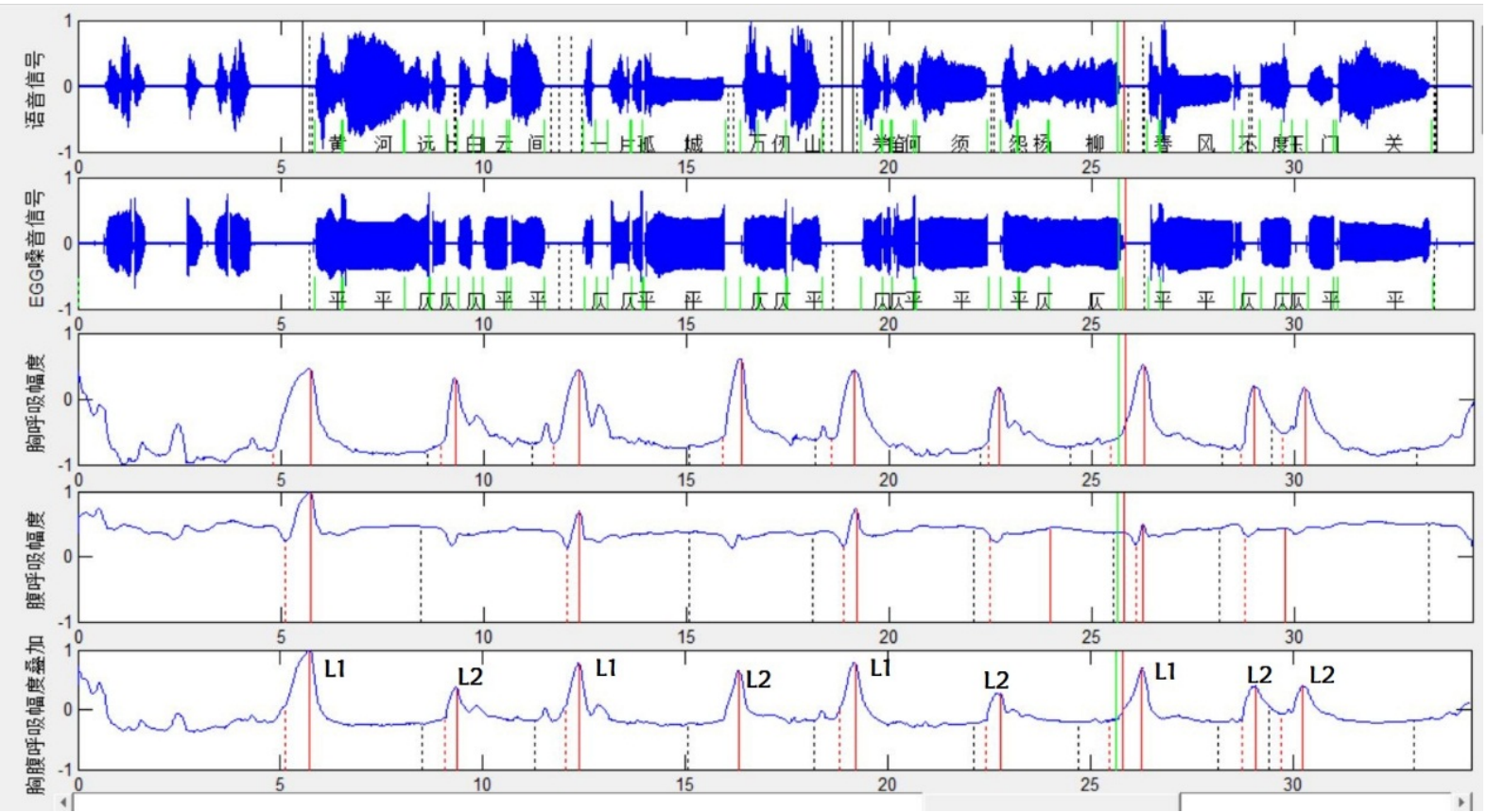

Figure 2. Comparison of chest and abdominal respiratory and phonetic signals in the poem of Liangzhou Ci (《凉州 词》).

As shown in Figure 2, the red dotted lines are meant to the start of the inspiration, the red solid lines are meant to the start of the expiration, and the black dotted lines represent the end of the respiration. Generally speaking, the chest and abdominal respiratory signals of chanting seven-syllable poems are distributed at two levels: At the beginning of a seven-syllable clause, there usually exists a first-level (L1) breath reset. Another breath reset, marked as second-level (L2), appears in the middle of clause.

By comparing with different poems with different Level-Oblique (平入) tone patterns, some interesting results are found in this research: (1) There is a L1 chest and abdominal breath reset at the beginning or the end of each clause, and a L2 breath reset appears inside the clause. (2) There is a close relationship between the position of the L2 breath reset and the Level-Oblique tone pattern. Except the poems with the tone pattern of Level start and Level end (平起平收) always having a L2 abdominal and chest breath reset in the end of the second syllable, all the seven-syllable poems with other tone patterns usually have a L2 abdominal and chest breath reset in the end of the fourth syllable of each clause when chanting in Cantonese. The example of Level start and Level end poem is showed in Figure 3. In Figure 3, a raw like Mei Feng Jia Jie Bei Si Qin (每逢佳节 倍思亲) of the poem named Jiu Yue Jiu Ri Yi Shan Dong Xiong Di (《九月九日忆山东兄弟》) is used as an example. After Mei Feng, there is a L2 abdominal breath and chest breath reset, in which the amplitude of expiratory reset is 0.58 and 0.37. In Figure 4, taking Level start and Level end (平起平收) poem of Wu Ti (《无 题》) as the example of other Level-Oblique tone patterns. A raw like Zuo Ye Xing Chen Zuo Ye Feng (昨夜星 辰昨夜风) in the poem of $W u T i$ is extracted as an example. After the last syllable of Zuo Ye Xing Chen, there is an obvious L2 chest breath reset, in which the amplitude of expiratory reset is 0.31 . 


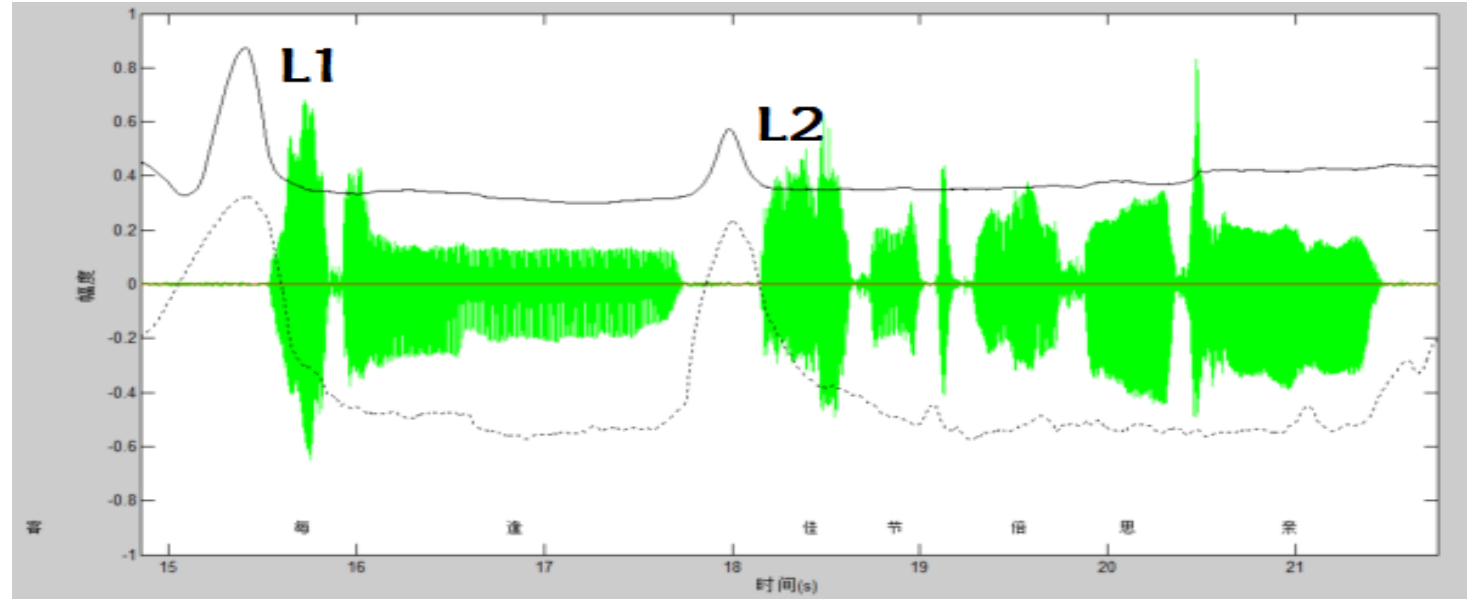

Figure 3. Comparison of chest and abdominal respiratory and phonetic signals on Level-start and Level-end tone pattern in the poem of Jiu Yue Jiu Ri Yi Shan Dong Xiong Di.

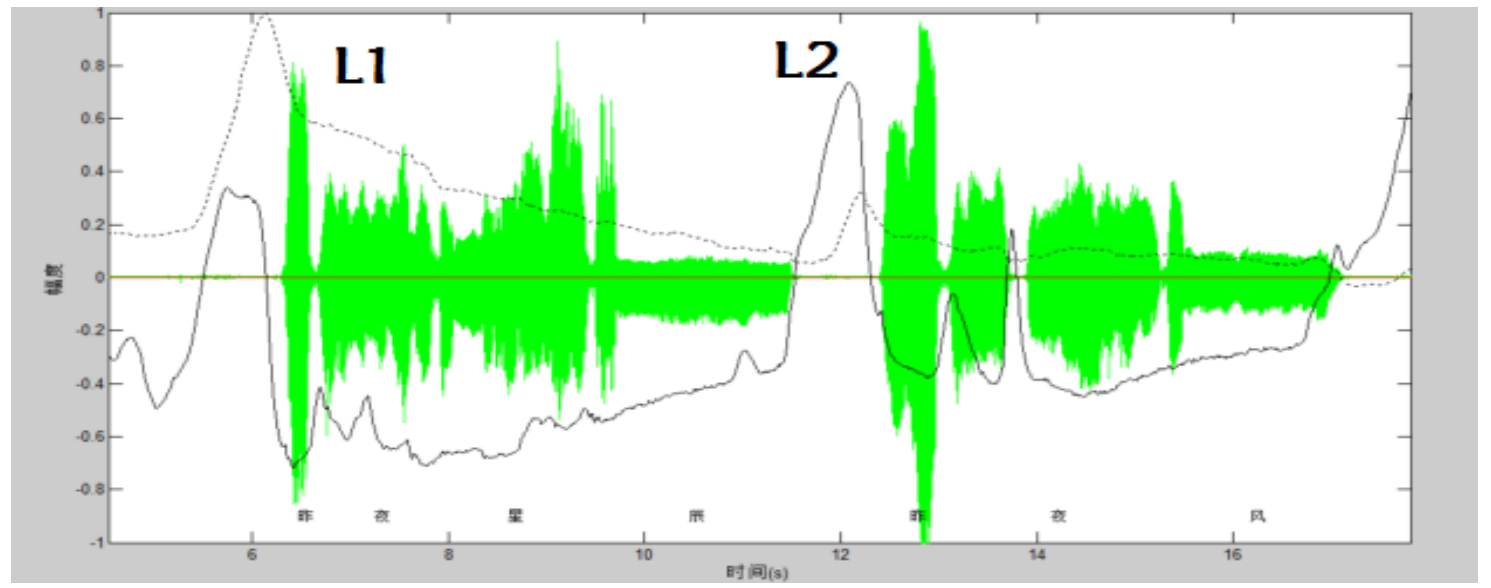

Figure 4. Comparison of chest and abdominal respiratory and phonetic signals on Oblique-start and Level-end tone pattern in the poem of Wu ti.

\section{The Relationship Between Respiratory Prosody and Phonetic Pause-Extension of Seven-Syllable Modern-Style Poems Chanted in Cantonese}

Yang Feng (2012) argued that distinguishing the boundary of prosodic clause and prosodic phrase is mainly by phonetic pause, so he used a way of phonetic pause clustering analysis to differentiate the boundary of different prosodic hierarchy. In this research we found that phonetic pause is not an ideal parameter to mark the boundary of prosodic phrase of chanting poems, and sometimes it is opposite to our knowledge. Taking Figure 5 as an example, the phonetic pause clustering analysis was used to analyse the prosodic phrase after each syllable in the Level-start and Level-end tone pattern of seven-syllable modern-style poems, and found that the phonetic pause duration behind the fourth syllable is the longest. If we take the end of the fourth syllable as the boundary of prosodic phrase of chanting poems, it would be contrary to our subjective knowledge (traditional chanting always emphasizes Long level and short oblique and the coda drawl) and respiratory prosody. So in this research, duration of pause-extension is used to determine the boundary of prosodic clause and prosodic phrase. 


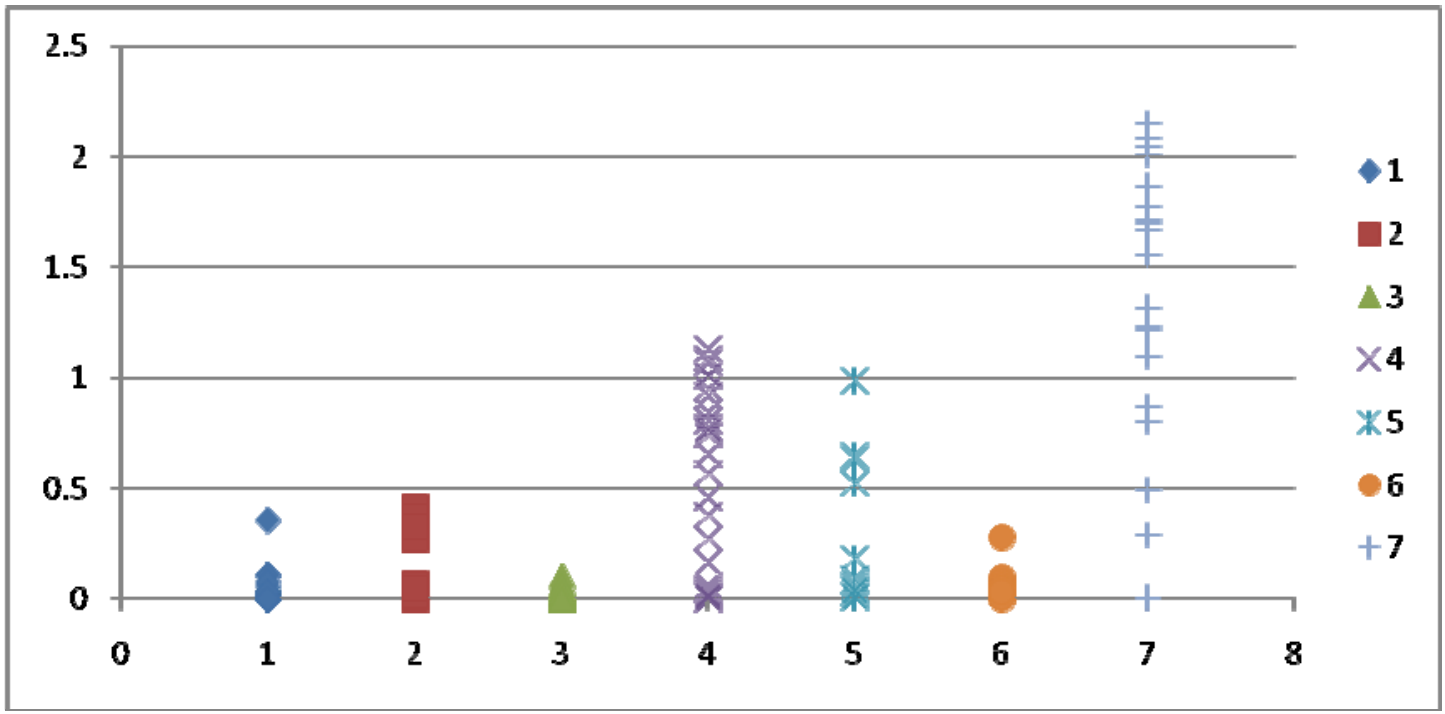

Figure 5. Syllable pause duration of the poem Wu ti with tone pattern of Level-start and Level-end.

Two phonetic prosodic hierarchies can be distinguished by the duration of pause-extension of syllables in the poems of chanting: (1) The longest pause-extension appears in the end of the last syllable of each clause, the average duration is about $1.37 \mathrm{~s}$, as we can see in Table 1. (2) The longer pause-extension appears in the middle of each clause and has a strong relation with tone patterns. For example, the longer pause-extension appears in the end of the second syllable of the clause with the tone pattern of Level-start and Level-end, the average duration is 1.28s, just as Figure 6 shows. While for the clause with other tone patterns, the longer pause-extension usually appears at the end of the fourth syllable. Taking Figure 7 as an example, the longer pause-extension appears at the end of the fourth syllable of the clause with the tone pattern of Oblique-start and Level-end (大起平收), and the average duration is 1.73s.

Table 1

Syllable Pause-Extension Average Duration of the Last Word of Each Sentence of Seven-Syllable Modern-Style Poems $^{1}$

\begin{tabular}{llllll}
\hline Last syllable position of & The first clause & The second clause & The third clause & The fourth clause $\begin{array}{l}\text { Average syllable duration of } \\
\text { the last clause }\end{array}$ \\
\hline $\begin{array}{l}\text { Average } \\
\text { duration }\end{array}$ & $1.03 \mathrm{~s}$ & $1.33 \mathrm{~s}$ & $1.21 \mathrm{~s}$ & $1.89 \mathrm{~s}$ & $1.37 \mathrm{~s}$ \\
\hline
\end{tabular}

\footnotetext{
1 There are eight clauses in the poem of Lv Shi (律诗) and four clauses in the poem of Jue Ju (绝句), for the convenience of comparison, only the last syllable pause-extension duration of the first four sentences were selected in the seven-character modern-style poems.
} 


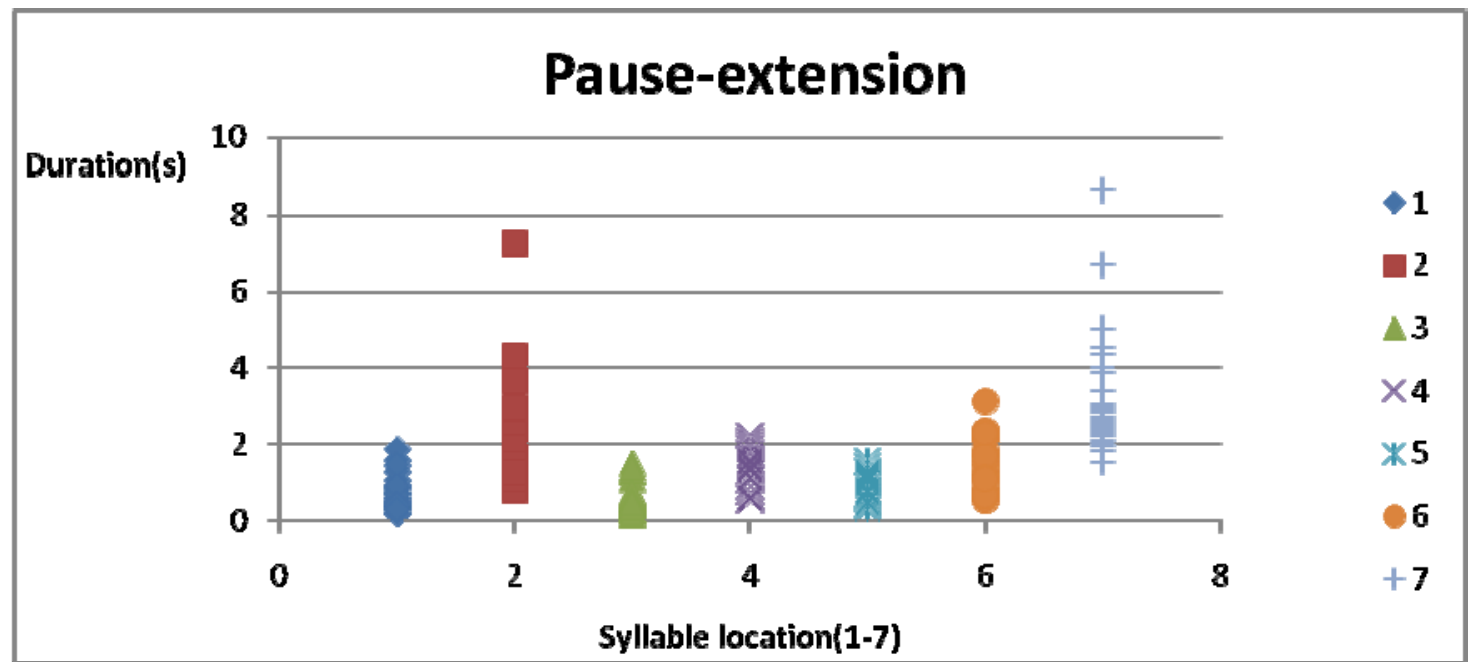

Figure 6. Syllable pause-extension duration of the clauses with tone pattern of Level-start and Level-end.

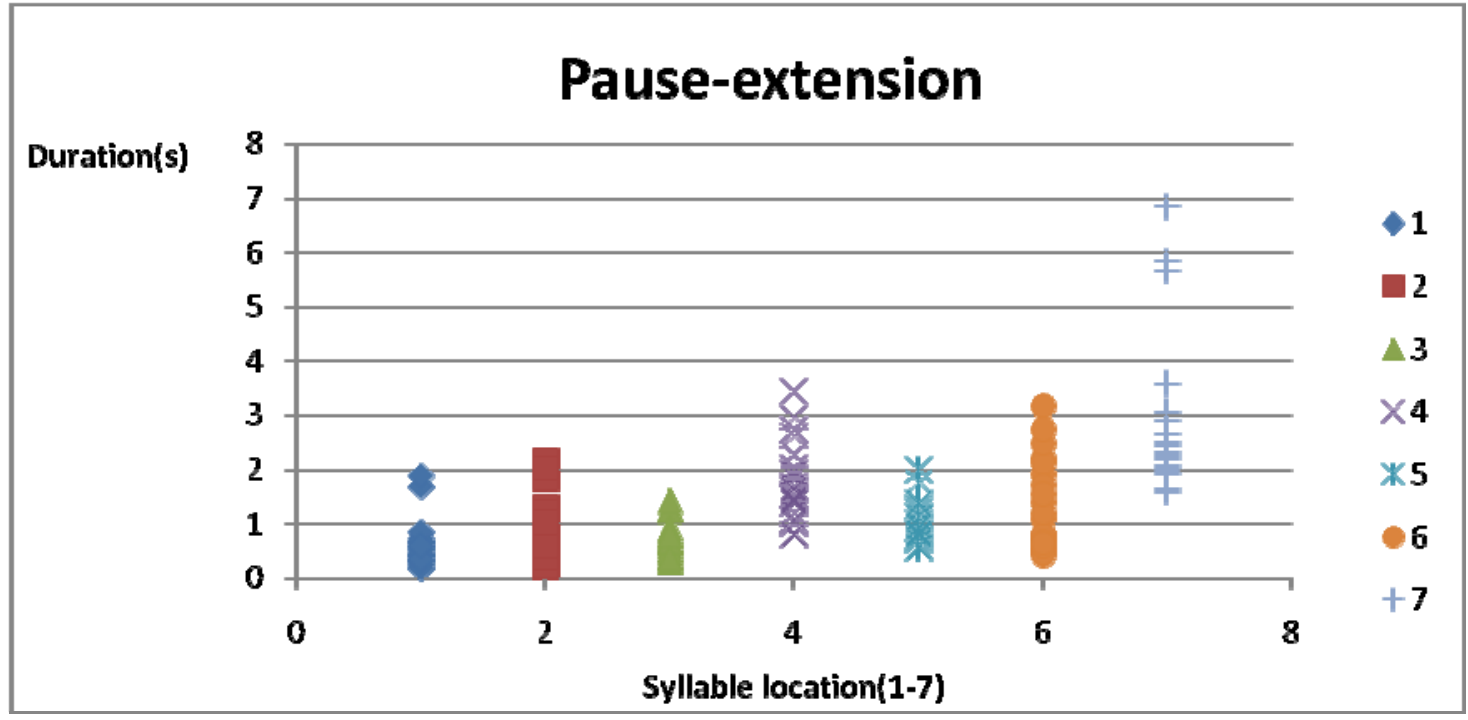

Figure 7. Syllable pause-extension duration of the clauses with tone pattern of Oblique-start and Level-end.

Comparing phonetic signals with respiratory signals, it is easy to conclude that there is a strong connection between phonetic signals and respiratory signals: L1 chest and abdominal breath reset relates with the longest pause-extension, always appears in the end of each clause. L2 chest and abdominal breath reset relates with the longer pause-extension, usually appear in the end of the second syllable of the clause with tone pattern of Level-start and Level-end, or at the end of the fourth syllable of the clause with other tone patterns.

\section{Conclusion}

In this research, chest and abdominal respiratory signals and phonetic signals of seven-syllable modern-style poems chanted in Cantonese were collected simultaneously by using the equipment of microphone, sound console, electroglottography, respiratory belt transducer, 16 channel collector and so on. The paper focuses on the respiratory prosody of seven-syllable modern-style poems chanted in Cantonese, especially the relationship between respiratory prosody and sentential pause-extension, and the following conclusions can be drawn: 
The research reveals a two-level chest and abdominal breath reset: L1 breath reset always appears at the beginning or the end of the clause; L2 breath reset usually appears in the middle of the clause, with the position relating to the tone patterns.

There is a strong relationship between phonetic signals and respiratory signals: L1 chest and abdominal breath reset relates with the longest pause-extension, always appearing at the end of each clause. While L2 chest and abdominal breath reset relates with the longer pause-extension, usually emerging at the end of the second syllable of the clause with tone pattern of Level-start and Level-end, or at the end of the fourth syllable of the clause with other tone patterns.

The parameter of phonetic pause-extension is better than phonetic pause to determine the prosodic hierarchy of seven-syllable modern-style poems in chanting. Comprehensive utilization of respiratory reset and phonetic pause-extension can better help us to determine the prosodic border of seven-syllable modern-style poems in chanting.

\section{References}

Hixon, T. J. (1987). Respiratory function in speech and song. San Diego: College-Hill Press.

Baken, R. J., Cavallo, S. A., \& Weissman, K. L. (1979). Chest wall movements prior to phonation. Journal of Speech and Hearing Research, 22, 862-872.

SHI, F., ZHANG, J. Y., BAI, X. J., \& ZHU, Z. H. (2010). Intonation and respiration: A preliminary analysis. Journal of Chinese Linguistics, 38(2), 323-335.

Slifka, J. (2000). Respiratory constraints on speech production at prosodic boundaries. Cambridge: Harvard-MIT.

YANG, F. (2012). Studies on Chinese traditional chanting—From perspectives of prosody, phonation and respiration (Ph.D. Thesis of Peking University).

YANG, F. (2015). A study on the features of chest and abdominal breathing between reciting and chanting Chinese poetry. Journal of Chinese Linguistics, 43(1B), 399-410.

YUAN, C., \& LI, A. J. (2007). The pause in expressive speech. NCMMSC, 195-201.

ZHANG, J. Y. (2011). The alternating relation between the speech prosody and the respiratory rhythm in Mandarin (Ph.D. Thesis of Nankai University).

ZHANG, J. Y., SHI, F., \& BAI, X. J. (2011). Preliminary analysis of respiratory diversity between the states of narrating and reading. Nankai Language Journal, 19(1), 56-63.

ZHAO, Y. R. (1994). Zhao Yuanren music collected papers. Beijing: China Wenlian Press. 